\title{
El debate de la Reforma Educativa: un caso de debate tecnocrático*
}

* Este artículo fue publicado originalmente en abril de 1972 en Revista de Ciencias de la Educación, año III, núm. 7, pp.57-58.
El debate acerca de las características de la Reforma Educativa que se viene implantando en nuestro país desde el gobierno de Onganía en adelante no produjo una abundante literatura. Sin embargo, los materiales periodísticos y algunos ensayos pretendidamente serios son una buena base para intentar una caracterización general del marco del debate, sus limitaciones y las raíces de esas limitaciones. El carácter de esta nota es meramente introductorio; no tiene la pretensión de abarcar la totalidad del problema, sino solamente señalar algunos puntos que pueden ser guía para un tratamiento y discusión más amplio.

El primer aspecto a tenerse en cuenta es el de los límites dentro de los cuales se encaró la discusión. Analíticamente, podríamos distinguir dos planos diferentes. El primero de ellos se refiere a la discusión de aspectos propiamente pedagógicos, tales como la justificación o no de la existencia de un nuevo nivel, la organización curricular y sus contenidos, la metodología general utilizada en el proceso de aprendizaje, etcétera. Este conjunto de problemas mereció un tratamiento abundante y algunos aportes alcanzaron un nivel destacable. El segundo de los planos de discusión mencionados es el sociopolítico, en el cual pueden agruparse los planteos referidos a las intenciones políticas de la Reforma y su relación con el desarrollo económico-social en general. Seguidamente intentaremos analizar aquellos ítems que -se nos ocurre-constituyen las principales limitaciones del debate en los dos planos mencionados.

\section{El nivel pedagógico}

En este nivel, toda reforma educativa aparece ante muchos educadores como la ocasión anhelada para llevar a la práctica del sistema educativo en su totalidad los principios científicos elaborados en los gabinetes y laboratorios experimentales. En este sentido, la reforma argentina no fue una excepción y muchos creyeron que -por fin- nuestro sistema educativo podría adecuarse a las exigencias de los últimos postulados pedagógicos en materia de métodos, contenidos, actitudes docentes, etcétera. Probablemente, el caso más significativo de esta ilusión lo constituya el tan citado ejemplo de la justificación de Víctor Mercante a la reforma de 1916 a través de las 437 páginas de su obra La Crisis de la Pubertad. En la actualidad, con muchos menos rigor y solidez que en 1916 y con 
las infaltables citas de las obras de Jean Piaget, muchos educadores se esforzaron por demostrar, por ejemplo, que la creación de un nuevo nivel se ajustaba a las hipótesis de la psicología genética, que la elevación del magisterio a nivel superior garantizaría la actualización metodológica y el cambio de actitudes tan necesarios en los docentes, etcétera. Sin embargo, no es nuestro propósito -y esto queremos destacarlo- discutir esos problemas en sí mismos, sino advertir que ni en 1916 el centro del debate estaba en la verdad de las curvas estadísticas de Mercante, ni en la actualidad está en las postulaciones de las teorías de la evolución psicológica. Y esto es así, en primer lugar, porque una Reforma es ante todo un hecho político, y el debate a niveles exclusivamente pedagógicos tiende a ocultar ese carácter.

Pero aquí no se trata meramente de una diferencia de enfoques igualmente válidos. Las limitaciones del nivel pedagógico de discusión plantea problemas muy serios y poco estudiados hasta ahora, que no se reducen al hecho obvio de que la educación abarca -como fenómeno- dimensiones sociales, políticas, económicas, etcétera, y que cada una de ellas tiene para sus problemas, sus propias explicaciones.

Las limitaciones del nivel pedagógico se ponen de manifiesto aún en las explicaciones de los aspectos más específicos de ese nivel, como puede ser -por ejemplo- todo el problema de la metodología de la enseñanza. Al respecto, la cuestión crucial que se plantea puede ser formulada en los términos siguientes: desde hace tiempo los científicos de la educación han elaborado una serie de postulados -traducidos en normas metodológicas, organizativas, etcétera- que se caracterizaron tanto por el énfasis puesto en la actividad del educando como por su impotencia para penetrar masivamente en los sistemas escolares. Con diferentes conceptualizaciones y mayor o menor rigor y coherencia, los planteos de una educación adaptada a las necesidades del educando, que promueva su actividad creadora y no su pasividad receptiva se repiten desde, por lo menos, Pestalozzi en adelante. Sin embargo, la aplicación de estos principios nunca fue más allá de ámbitos reducidos, marginales al sistema educativo masivo. Este hecho plantea una serie de interrogantes cuya respuesta parece trascender el marco de los métodos mismos para afectar a la estructura social en su conjunto y a la educación como institución particular de esa estructura. Una posible explicación -planteada aquí a título de hipótesis- radicaría en que la resistencia de la educación institucionalizada para incorporar la nueva metodología se debe a que los postulados sobre los que ella se asienta entran en contradicción con la función social de la educación en general. De ahí que no sea casual el hecho de que las experiencias más significativas de aplicación a niveles masivos del sistema escolar de los principios metodológicos activos se hayan dado en países que pasaban por procesos de cambios revolucionarios acelerados; el caso típico podría representarlo la U.R.S.S. en los años inmediatamente posteriores a la revolución de 1917 hasta el advenimiento del stalinismo, que retrotrajo la situación pedagógica (métodos, actitudes, etcétera) a prácticas propiamente tradicionales.

Si se admite este planteo, la primera consecuencia que puede extraerse es que la importancia de la variable sociopolítica para la consideración de los problemas didácticos en general es más relevante que lo que se presumía implícitamente hasta ahora. Muy sintéticamente, esto puede ser formulado en los términos de Bourdieu y Passeron (1970), diciendo que la educación adecua su funcionamiento interno al cumplimiento de su función externa: función social de conservación y de reproducción del orden existente.

Pero la importancia señalada más arriba adquiere su máxima tonalidad cuando se trata precisamente de la discusión de procesos de reformas educativas a nivel de los sistemas escolares. La conexión aquí planteada entre metodología y realidad sociopolítica tira abajo las ilusiones de muchos educadores y abre una nueva perspectiva de análisis para los problemas didácticos, cuyas primeras expresiones recién ahora se están produciendo. Sin embargo, el debate de la actual reforma no rozó ni siquiera la superficie 
de estos problemas. La característica más saliente -que se repite en la discusión del nivel sociopolítico- fue la abstracción de las relaciones sociales en su conjunto dentro de las cuales se opera una reforma educativa. El ejemplo más claro de esta abstracción se advierte en el tratamiento del problema de la orientación, problema considerado fundamental en la discusión sobre la implantación del nivel intermedio. Ni partidarios ni opositores cuestionaron la legitimidad de la implantación dentro del sistema educativo de ciertos mecanismos de orientación que garantizasen un aprovechamiento racional de los recursos humanos. El debate, en cambio, giró en torno a cómo y cuándo incorporar esos mecanismos. Sin embargo, una discusión planteada en esos términos es típicamente un caso de debate tecnocrático donde las relaciones de clase (clases sociales) están totalmente excluidas. Planteado en términos muy directos, esa exclusión lleva a dejar de lado el problema de la incidencia diferencial de esos mecanismos según las distintas clases sociales. En otras palabras, que a las profesiones de menor prestigio, sin proyecciones futuras, es decir, de menor rango dentro del sistema, serán "orientados" los alumnos provenientes de clases sociales populares. La verdad de esta proposición se puede corroborar con solo analizar los datos disponibles sobre el origen social de los alumnos de las diferentes modalidades de los sistemas escolares "modernos" que poseen los países capitalistas más desarrollados.

\section{El nivel sociopolítico}

La justificación (y gran parte de los ataques) de la Reforma Educativa desde el nivel sociopolítico de discusión estuvo centrada en el postulado de la necesidad de adecuar el sistema educativo a las necesidades del desarrollo económico y, en general, de una sociedad en proceso de cambio.

Estos planteos, que configuran una concepción que bien podría denominarse desarrollismo ingenuo, estaban apoyados en la creencia de que la educación juega un papel crucial en el proceso de desarrollo económico y que la única exigencia de la que debe hacerse cargo es la de promover carreras técnico-profesionales según las necesidades de un mercado ocupacional pretendidamente escaso en esas ramas.

Hace tiempo, sin embargo, que los científicos sociales que se ocupan de este tipo de problemas en América Latina han planteado que -al colocar esa relación en el marco de los intereses de clase internos de cada nación y de las relaciones de dominación a nivel internacional- los problemas de la educación y del desarrollo económico se complejizan y se redimensionan.

Pero no es el objetivo de esta nota repetir esos planteos. Preferimos, en cambio, continuar con lo expuesto en el parágrafo anterior y señalar, aquí también, el carácter tecnocrático de la discusión. En este nivel, la abstracción de las condiciones políticas en las cuales se enmarca la acción educativa tiene dos tipos de expresiones. Una de ellas, muy burda, es la expuesta por algunos partidarios del "cambio" y del "desarrollo" en general. Así, por ejemplo, se ha sostenido que:

La virtud del proceso reiniciado es que, esencialmente, representa el clima y el cauce para una actitud renovadora. Y esta actitud debe salvarse, consolidarse -llevada hasta sus últimas consecuencias positivas-, cualquiera sea el titular del poder y las contingencias que advengan en el camino político. (Salonia, 1971)

Además, existe otra expresión más sutil de esta forma de pensar, la cual -por ello- es característica a su vez de vastos sectores opositores a la Reforma, que limitan su cuestionamiento a este gobierno, pero no al sistema en general. Esta otra forma es precisamente 
la que expresa el modo de pensamiento tecnocrático en su alcance más general. Admite como axioma básico la necesidad de adecuar la educación a las necesidades del sistema económico, pero -apoyándonos nuevamente en Bourdieu y Passeron- no advierten que hacer esto implica reintroducir las otras funciones de la educación (funciones sociales) bajo la apariencia de funciones técnicas. Esto solo es posible omitiendo relacionar con una determinada estructura de clases el sistema económico al cual se subordina el sistema de enseñanza y considerando obvia una demanda económica concebida como independiente de las relaciones de fuerza entre las clases (Bourdieu y Passeron, 1970). 


\section{Q Referencias bibliográficas}

» Bourdieu, P. y Passeron, J. C. (1970). La Reproduction. París, Minuit.

»Salonia, A. (1971). El último tramo de la educación básica. En Villaverde, A. (dir.), La Escuela Intermedia en Debate, pp. 125. Buenos Aires, Humanitas. 
